\title{
The Population of Fusarium oxysporum f. sp. fragariae, Cause of Fusarium Wilt of Strawberry, in California
}

P. M. Henry, S. C. Kirkpatrick, C. M. Islas, A. M. Pastrana, and J. A. Yoshisato, Department of Plant Pathology, University of California, Davis 95616; S. T. Koike, University of California Cooperative Extension, Salinas 93901; O. Daugovish, University of California Cooperative Extension, Ventura 93003; and T. R. Gordon, Department of Plant Pathology, University of California, Davis

\begin{abstract}
The objectives of this study were to investigate the structure of the population of Fusarium oxysporum f. sp. fragariae in California and to evaluate methods for its detection. Fifty-nine isolates of $F$. oxysporum f. sp. fragariae were obtained from diseased strawberry plants and their identity was confirmed by pathogenicity testing. The full nuclear ribosomal intergenic spacer (IGS) and elongation factor 1- $\alpha$ gene (EF-1 $\alpha$ ) were amplified by polymerase chain reaction (PCR) and sequenced to elucidate phylogenetic relationships among isolates. IGS and EF-1 $\alpha$ sequences revealed three main lineages, which corresponded to three somatic compatibility groups. Primers designed to detect $F$. oxysporum f. sp.

fragariae in Japan amplified a 239-bp product from 55 of 59 California isolates of $F$. oxysporum $\mathrm{f}$. sp. fragariae and from no nonpathogenic isolates of $F$. oxysporum. The sequence of this PCR product was identical to the sequence obtained from $F$. oxysporum $\mathrm{f}$. sp. fragariae isolates in Japan. Intensive sampling at two locations in California showed results of tests based on PCR and somatic compatibility to be in agreement for $97 \%$ ( 257 of 264) of isolates tested. Our findings revealed considerable diversity in the California population of $F$. oxysporum $\mathrm{f}$. sp. fragariae, and indications that horizontal gene transfer may have occurred.
\end{abstract}

Fusarium wilt, caused by Fusarium oxysporum f. sp. fragariae, is an important disease of strawberry (Fragaria $\times$ ananassa) that affects production of this crop in many locations throughout the world (Koike and Gordon 2015). The pathogen resides in soil, infects roots, and grows into the xylem, which results in a systemic infection. Pathogen growth in the xylem and the plant's response impede the flow of water to the shoot, which contributes to development of symptoms, including stunting, wilting, and dieback. In heavily infested fields, plants may be severely stunted early in the season and produce no fruit (Koike and Gordon 2015).

Fusarium wilt of strawberry was first described in Australia in 1962 (Winks and Williams 1965) and was shortly thereafter reported to occur in Japan (Okamoto et al. 1970). This disease was identified in California in 2006, in fields where flat fumigation with methyl bromide and chloropicrin was no longer used (Koike et al. 2009). Though initially restricted to isolated patches in a few locations, by 2009 Fusarium wilt was causing extensive damage to numerous fruit production fields in southern California. Fusarium wilt is now found in all major strawberry production areas in California, and constitutes a significant threat to this $\$ 2.6$ billion industry, which produces over $80 \%$ of the fresh strawberry fruit consumed in the United States (California Department of Food and Agriculture 2014; USDA Economic Research Service 2015).

For many crops, control of Fusarium wilt has been achieved through the use of disease-resistant cultivars (Cianchetta and Davis 2015; Gordon et al. 1990; McGovern 2015). This approach will become increasingly important for management of soilborne diseases of strawberry, including Fusarium wilt, as environmental regulations become more restrictive, limiting options for chemical control (Koike and Gordon 2015). Breeding for resistance to disease requires identification of appropriate pathogen isolates to be included in a screening procedure. A useful step in the isolate selection process is an assessment of genetic diversity in the pathogen population within the region where the crop is grown. To this end, isolates of $F$. oxysporum f. sp. fragariae obtained from throughout major strawberry production areas in California were characterized by (i) an assessment of interisolate

Corresponding author: T. R. Gordon; E-mail: trgordon@ucdavis.edu

Accepted for publication 25 October 2016.

() 2017 The American Phytopathological Society somatic compatibility, (ii) sequencing of the elongation factor $1-\alpha$ gene $(\mathrm{EF}-1 \alpha)$ and the nuclear ribosomal intergenic spacer (IGS), and (iii) testing for the presence of a diagnostic amplicon using a polymerase chain reaction (PCR) test developed by Suga et al. (2013). The objectives of this work were to determine whether the California population of $F$. oxysporum f. sp. fragariae included distinctive genetic subgroups and to evaluate the correspondence between results of the PCR test (Suga et al. 2013), somatic compatibility, and pathogenicity to strawberry.

\section{Materials and Methods}

Isolates. Isolates of $F$. oxysporum were obtained from symptomatic strawberry plants in commercial fruit production fields throughout California. Tissue segments taken from crowns or petioles were briefly dipped in $70 \%$ ethanol, submersed in $1 \%$ sodium hypochlorite for $2 \mathrm{~min}$, and cultured on plates of Komada's selective medium (KM) (Komada 1975) at room temperature under continuous fluorescent light (24 h/day). Spores removed from colonies identified as $F$. oxysporum on KM were streaked on water agar, and single-spore subcultures were stored on dried filter paper (Gordon and Okamoto 1991). Each isolate was cultured on KM and, after 7 to 10 days of growth at room temperature under continuous fluorescent light $(24 \mathrm{~h} /$ day $)$, colonies were provisionally identified as $F$. oxysporum $\mathrm{f}$. sp. fragariae based on colony morphology: fluffy, white, aerial mycelium, tight colony margins, and light pink to orange coloration on the underside of the colony. Additional isolates of $F$. oxysporum were obtained from naturally infested field soils using dilution plating, as described by Scott et al. (2010), and cultured on KM as described above. Isolates of $F$. oxysporum f. sp. fragariae from Japan (MAFF712071, MAFF727510, and MAFF744009) were kindly provided by the National Institute of Agrobiological Sciences in Japan. All isolates used in this study are maintained in a culture collection in the Department of Plant Pathology at University of California (UC) Davis, and are available on request from the corresponding author.

Pathogenicity tests. Inoculum was obtained by growing isolates on plates of potato dextrose agar (PDA) at room temperature under continuous fluorescent light for 3 to 4 weeks and collecting spores as described by Schmale and Gordon (2003). To test for pathogenicity, roots of 'Albion' strawberry plants were dipped in an aqueous suspension of $5 \times 10^{6} \mathrm{spores} / \mathrm{ml}$ for $7 \mathrm{~min}$, transplanted into 2.0 -liter pots filled with Sunshine Mix 1 (Sun Gro Horticulture), and maintained in a controlled environment chamber with a 12 -h photoperiod and day and night temperatures of 25 and $18^{\circ} \mathrm{C}$, respectively. Four plants were inoculated with each isolate. Six to eight weeks after inoculation, plants were 
judged to be diseased if they manifested symptoms that included stunting, yellowing of leaves, wilting, and, in some cases, death. Petioles and crowns were cultured as described above to confirm recovery of $F$. oxysporum. Each pathogenicity test included control plants that were treated in the same manner as inoculated plants except that roots were dipped in water instead of a spore suspension. In all cases, control plants remained healthy for the entire observation period.

Somatic compatibility tests. Interisolate somatic compatibility was assessed using nitrate nonutilizing (nit) mutants, as described by Jacobson and Gordon (1988). The nit mutants were obtained by culturing wild-type isolates on PDA amended with $4 \%$ potassium chlorate. Rapidly growing sectors were judged to be resistant to chlorate and transferred to Czapek solution agar (CZ), in which nitrate is the sole supplemental source of nitrogen. Colonies manifesting flat growth (no aerial mycelium) on $\mathrm{CZ}$ were identified as nit mutants. Complementary nit phenotypes, differentiated as described by Correll et al. (1987), were obtained for a single isolate of $F$. oxysporum $\mathrm{f}$. sp. fragariae to serve as tester strains. The nit mutants obtained from other isolates were paired with tester strains on $\mathrm{CZ}$. Where aerial mycelium, indicative of wild-type growth, developed along the line of contact between the unknown isolate and one or both of the tester nit mutants, that isolate was assigned to the somatic compatibility group (SCG) represented by the testers. The absence of wild-type growth indicated that an isolate was not compatible with the tester strains (Jacobson and Gordon 1988). When this occurred, complementary nit mutants were obtained from the incompatible isolate to serve as tester strains for a new SCG. This process was continued until all isolates were associated with an SCG.

DNA extraction and PCR. Isolates were grown on PDA for 7 days to obtain fungal biomass. Aerial mycelia were scraped from the surface of the agar, and DNA was extracted using the OmniGen-X Purespin gDNA Purification Kit per the manufacturer's instructions, with the minor modification that cell lysis on a vortex was conducted for 15 min instead of 10 . DNA concentration was determined on a ND-1000 NanoDrop spectrophotometer (NanoDrop Products). Regions to be sequenced were obtained using PCR on an Applied Biosystems 2720 thermal cycler. The EF- $1 \alpha$ gene was amplified with primers (EF1 and EF2) using the protocol described by O'Donnell et al. (1998). The IGS, which separates repeat units of tandem arrays of rDNA genes, was amplified with primer pairs iNL11/CNSa and iCNS11/NLa using the reaction mixture and parameters described by O'Donnell et al. (2009). A sequence reported to be diagnostic for $F$. oxysporum f. sp. fragariae in Japan was amplified with the FofraF/FofraR primer pair using the protocol described by Suga et al. (2013). PCR products were visualized with EZ Vision Three DNA dye (VWR International) on agarose gels containing $1 \%$ agarose in $1 \times$ Tris-acetate EDTA buffer.

DNA sequencing and alignment. PCR products were purified with the Bioneer Accuprep PCR Purification Kit per the manufacturer's instructions and sequenced using BigDye Terminator v3.1 Cycle Sequencing Kit with Gel Company Better Buffer at the UC Davis DNA Sequencing Facility. Raw reads were assembled into consensus sequences in BioEdit version 7.2.5 (http://www.mbio.ncsu.edu/BioEdit/ bioedit.html) and aligned in MEGA6 using ClustalW (Larkin et al. 2007; Tamura et al. 2013). GenBank accession codes for California isolates of $F$. oxysporum $\mathrm{f}$. sp. fragariae are listed in Table 1 . Accession codes for sequences obtained from other isolates used in this study are GL1702 (IGS: KX451272, EF-1 $\alpha$ : KX456103), GL1703 (IGS: KX451282, EF-1 $\alpha$ : KX456104), GL1704 (IGS: KX451283, EF-1 $\alpha$ : KX456105), MAFF712071 (IGS: KX451276, EF-1 $\alpha$ : KX456038), MAFF727510 (IGS: KX451277, EF-1 $\alpha$ : KX456039), and MAFF744009 (IGS: KX451278, EF-1 $\alpha$ : KX456040). Accession codes for $\mathrm{EF}-1 \alpha$ sequences from other studies that were included in our data set are ATCC18138 (KX456102), G2-4 (KX456099), FPOST140 (KX456101), MAFF305557 (KX592590), 15-8KFs (KJ776747), 16C-30KFsa (KJ776746), 18-13KFs (KJ776745), 23c-12KFsa, (KJ776744), NRRL26438 (FJ985295), RBG5771 (KJ397042), RBG5791 (KJ397057), RBG5814 (KJ397069), RBG5782 (KJ397051), RBG5781 (KJ397050), RBG5769 (KJ397041), RBG5796 (KJ397061), RBG5801 (KJ397062), and RBG5803 (KJ397064) (Dinler et al. 2016; Laurence et al. 2014; O’Donnell et al. 2009; Pastrana 2014).
Phylogenetic analysis. Phylogenetic trees were constructed in PAUP* v4.0a145 using maximum-likelihood (ML) and using maximum-parsimony (MP) analyses with 10,000 bootstrap replicates (Swofford 2002). For all trees, heuristic searches were performed with the tree bisection and reconstruction (reconnection limit $=8$ ) branchswapping algorithm. All characters were included, treated with equal weight, and assumed to be unordered. Gaps were treated as missing data and the Hasegawa-Kishino-Yano model was used to estimate transition/ transversion bias (Hasegawa et al. 1985). EF-1 $\alpha$ (GenBank accession AF160262) and IGS (GenBank accession AY249379) sequences from $F$. verticillioides NRRL 22172 served as outgroups in all trees (Herron et al. 2015; Schweigkofler et al. 2004). Compatibility of the EF-1 $\alpha$ and IGS datasets was evaluated by a partition homogeneity test implemented in PAUP*. Nodes with bootstrap support higher than $50 \%$ were included in the final trees. Phylograms were created for EF-1 $\alpha$ sequences and a concatenated dataset that included sequence data from both IGS and EF-1 $\alpha$, and deposited in TreeBASE under accession code S19538.

Efficacy of PCR and SCG detection methods. Naturally infested fields in Monterey and Ventura Counties were selected for intensive sampling of local populations of $F$. oxysporum $\mathrm{f}$. sp. fragariae. These two isolate collections were used to evaluate the association between SCG, the presence of a diagnostic DNA sequence (Suga et al. 2013), and pathogenicity to strawberry. At the Monterey County location, isolates of $F$. oxysporum were obtained from soil by dilution plating, as described above. Isolates deemed likely to be $F$. oxysporum f. sp. fragariae based on colony morphology were subcultured as single spores and tested for compatibility with tester strains representative of SCG identified from the statewide collection described above. Each isolate was also tested for the presence or absence of the diagnostic DNA sequence described by Suga et al. (2013). Isolates for which PCR and SCG results were not in accord were tested for pathogenicity, as described above.

For the Ventura County location, F. oxysporum f. sp. fragariae isolates were recovered from roots of strawberry plants grown in infested soil. Dormant crowns of Albion strawberry were transplanted into a 60:40 blend of sand (sterilized by autoclaving twice $24 \mathrm{~h}$ apart) and naturally infested field soil. The sand/soil mix was dispensed into 3.8-liter pots, and two strawberry crowns were placed at the center of each pot. Plants were maintained in a controlled environment chamber with a 12-h photoperiod and day and night temperatures of 28 and $20^{\circ} \mathrm{C}$, respectively. Between 2 and 3 weeks after planting, young feeder roots were separated from the soil matrix and transferred to a flask containing sterile $1 \%$ sodium hexametaphosphate, which serves to disperse soil aggregates into their component particles (Kettler et al. 2001). This flask was placed on a rotary shaker set at $50 \mathrm{rpm}$ for $15 \mathrm{~min}$, after which liquid was drained from the flask and replaced with fresh, sterile, $1 \%$ sodium hexametaphosphate. The flask was returned to the shaker for an additional $15 \mathrm{~min}$, followed by a third rinse using the same procedure, and a final rinse in sterile water. Thereafter, root tips, 0.5 to $2.5 \mathrm{~mm}$ in length, were placed on plates of $\mathrm{KM}$, which were incubated as described above. After 5 to 7 days, single hyphal tips of colonies provisionally identified as $F$. oxysporum $\mathrm{f}$. sp. fragariae based on colony morphology were transferred to PDA and tested for somatic compatibility and presence or absence of the diagnostic DNA sequence, as described above. Isolates for which PCR and SCG results were not in accord were tested for pathogenicity on strawberry, as described above.

\section{Results}

Isolate characterization. In total, 115 isolates of Fusarium oxysporum were obtained from diseased strawberry plants between 2008 and 2014 and tested for pathogenicity. Of these, 59 were confirmed to be pathogenic to strawberry, producing symptoms that ranged from stunting and leaf discoloration to severe wilting and death. F. oxysporum was reisolated from crowns and petioles of all symptomatic plants. In all, 54 isolates were associated with a single compatibility group (SCG 1), whereas the remaining 5 isolates were associated either with SCG 2 or SCG 3 (Table 1). No Japanese isolates of $F$. oxysporum $\mathrm{f}$. sp. fragariae were compatible with testers for a California SCG. The largest number of isolates $(n=31)$ was from Ventura County and included all three SCGs of 
Table 1. Somatic compatibility, presence or absence of a diagnostic DNA sequence, and GenBank accession codes for California isolates of Fusarium oxysporum f. sp. fragariae

\begin{tabular}{|c|c|c|c|c|c|c|}
\hline Isolate & Year $^{\mathbf{a}}$ & Location $^{b}$ & SCG $^{c}$ & $\mathbf{P C R}^{\mathbf{d}}$ & $E F-1 \alpha^{e}$ & $\mathbf{I G S}^{\mathbf{f}}$ \\
\hline GL0878 & 2008 & Ventura & SCG1 & + & KX456100 & KX451269 \\
\hline GL912 & 2012 & Monterey & SCG1 & + & KX456045 & NT \\
\hline GL945 & 2012 & Strawberry & SCG1 & + & KX456044 & NT \\
\hline GL952 & 2012 & Monterey & SCG1 & + & KX456043 & NT \\
\hline GL980 & 2013 & Ventura & SCG1 & + & KX456042 & NT \\
\hline GL991 & 2013 & Santa Cruz & SCG1 & + & KX456041 & NT \\
\hline GL1080 & 2012 & Ventura & SCG1 & + & KX456097 & KX451271 \\
\hline GL1081 & 2008 & Ventura & SCG1 & + & KX456096 & NT \\
\hline GL1144 & 2009 & Ventura & SCG1 & + & KX456095 & NT \\
\hline GL1147 & 2009 & Ventura & SCG1 & + & KX456094 & NT \\
\hline GL1148 & 2009 & Ventura & SCG1 & + & KX456093 & NT \\
\hline GL1149 & 2009 & Ventura & SCG1 & + & KX456092 & NT \\
\hline GL1150 & 2009 & Ventura & SCG1 & + & KX456091 & NT \\
\hline GL1154 & 2012 & Monterey & SCG1 & + & KX456090 & NT \\
\hline GL1155 & 2009 & Monterey & SCG1 & + & KX456089 & NT \\
\hline GL1156 & 2009 & Monterey & SCG1 & + & KX456088 & NT \\
\hline GL1157 & 2012 & Monterey & SCG1 & + & KX456087 & NT \\
\hline GL1160 & 2012 & Ventura & SCG1 & + & KX456086 & NT \\
\hline GL1162 & 2009 & Ventura & SCG1 & + & KX456085 & KX451273 \\
\hline GL1164 & 2009 & Ventura & SCG1 & + & KX456084 & NT \\
\hline GL1200 & 2010 & Ventura & SCG1 & + & KX456083 & NT \\
\hline GL1201 & 2010 & Ventura & SCG1 & + & KX456082 & NT \\
\hline GL1206 & 2010 & Ventura & SCG1 & + & KX456081 & NT \\
\hline GL1208 & 2010 & Ventura & SCG1 & + & KX456080 & NT \\
\hline GL1209 & 2010 & Ventura & SCG1 & + & KX456079 & NT \\
\hline GL1250 & 2011 & Ventura & SCG1 & + & KX456078 & NT \\
\hline GL1251 & 2011 & Ventura & SCG1 & + & KX456077 & NT \\
\hline GL1252 & 2011 & Ventura & SCG1 & + & KX456076 & NT \\
\hline GL1253 & 2011 & Ventura & SCG1 & + & KX456075 & NT \\
\hline GL1259 & 2011 & Monterey & SCG1 & + & KX456074 & NT \\
\hline GL1260 & 2011 & Monterey & SCG1 & + & KX456073 & NT \\
\hline GL1261 & 2011 & Monterey & SCG1 & + & KX456072 & NT \\
\hline GL1262 & 2011 & Monterey & SCG1 & + & KX456071 & NT \\
\hline GL1263 & 2011 & Monterey & SCG1 & + & KX456070 & NT \\
\hline GL1264 & 2011 & Monterey & SCG1 & + & KX456069 & NT \\
\hline GL1287 & 2011 & Ventura & SCG1 & + & KX456066 & NT \\
\hline GL1292 & 2011 & Ventura & SCG1 & + & KX456065 & NT \\
\hline GL1349 & 2012 & Monterey & SCG1 & + & KX456063 & NT \\
\hline GL1351 & 2012 & Monterey & SCG1 & + & KX456062 & NT \\
\hline GL1353 & 2012 & Monterey & SCG1 & + & KX456061 & NT \\
\hline GL1394 & 2013 & Ventura & SCG1 & + & KX456058 & NT \\
\hline GL1395 & 2013 & Ventura & SCG1 & + & KX456057 & NT \\
\hline GL1404 & 2014 & Santa Barbara & SCG1 & + & KX456056 & NT \\
\hline GL1405 & 2014 & Santa Barbara & SCG1 & + & KX456055 & NT \\
\hline GL1481 & 2014 & Monterey & SCG1 & + & KX456054 & NT \\
\hline GL1482 & 2014 & Monterey & SCG1 & + & KX456053 & NT \\
\hline GL1483 & 2014 & Monterey & SCG1 & + & KX456052 & NT \\
\hline GL1484 & 2014 & Monterey & SCG1 & + & KX456051 & NT \\
\hline GL1485 & 2014 & Monterey & SCG1 & + & KX456050 & NT \\
\hline GL1486 & 2014 & Monterey & SCG1 & + & KX456049 & NT \\
\hline GL1512 & 2014 & Ventura & SCG1 & + & KX456048 & NT \\
\hline GL1513 & 2014 & Ventura & SCG1 & + & KX456047 & NT \\
\hline GL1514 & 2014 & Ventura & SCG1 & + & KX456046 & NT \\
\hline GL1385 & 2012 & Santa Barbara & SCG1 & - & KX456059 & KX451281 \\
\hline GL1059 & 2008 & Ventura & SCG2 & - & KX456098 & KX451270 \\
\hline GL1270 & 2011 & Ventura & SCG2 & - & KX456067 & KX451275 \\
\hline GL1381 & 2012 & San Luis Obispo & SCG2 & - & KX456060 & KX451280 \\
\hline GL1268 & 2011 & Ventura & SCG3 & + & KX456068 & KX451274 \\
\hline GL1315 & 2011 & Santa Barbara & SCG3 & + & KX456064 & KX451279 \\
\hline
\end{tabular}

\footnotetext{
a Year isolate was obtained.

${ }^{\mathrm{b}}$ County in California: location of the field from which the isolate was obtained.

c Somatic compatibility group (SCG) with which the isolate was associated.

${ }^{\mathrm{d}}$ Polymerase chain reaction (PCR); a 239-bp amplicon was (+) or was not (-) obtained using the FofraF/FofraR primer pair.

e GenBank accession codes for elongation factor $1-\alpha$ gene $(\mathrm{EF}-1 \alpha)$ sequences.

${ }^{\mathrm{f}}$ GenBank accession codes for intergenic spacer (IGS) sequences; NT = isolates whose IGS sequence was not obtained.
} 
F. oxysporum f. sp. fragariae. Two SCGs were found in Santa Barbara County and one each in Monterey, Santa Cruz, and San Luis Obispo Counties (Table 2). Although subtle differences in colony morphology were observed between isolates associated with SCG 1, 2, and 3, these differences were not consistent enough to be useful for diagnostic purposes.

Primers developed for detection of $F$. oxysporum $\mathrm{f}$. sp. fragariae in Japan were tested on all 59 California isolates confirmed to be $F$. oxysporum f. sp. fragariae and 56 California isolates of $F$. oxysporum that were not pathogenic to strawberry. An amplicon of the expected size $(239 \mathrm{bp})$ was obtained from both isolates associated with SCG 3 and all but one isolate (GL1385) associated with SCG 1 (Table 1). No amplicon was obtained from any of the three $F$. oxysporum f. sp. fragariae isolates associated with SCG 2 or from any nonpathogenic isolate. Many different PCR cycle conditions and permutations of the reaction mixture were tested on nonamplifying isolates of $F$. oxysporum f. sp. fragariae but amplification was not observed. Sequencing of PCR amplicons obtained from GL1080, GL1162, GL1315, and GL1482 revealed 100\% sequence identity to that which was published by Suga et al. (2013).

Phylogenetic analysis. The EF- $1 \alpha$ locus was sequenced for 66 isolates of $F$. oxysporum f. sp. fragariae -59 from California (Table 1), 4 from Japan, 2 from Spain, and 1 from Australia-and 3 nonpathogenic isolates of $F$. oxysporum obtained from strawberry plants in California. All SCG 1 isolates had an identical EF-1 $\alpha$ sequence. Isolates associated with SCG 2 and SCG 3 shared a common EF-1 $\alpha$ sequence that differed from that of SCG 1 by $2 \mathrm{bp}$. Because sequences were the same within an SCG, a representative isolate was chosen from each California SCG for comparison with EF-1 $\alpha$ sequences from isolates of $F$. oxysporum $\mathrm{f}$. sp. fragariae originating in Spain, Australia, Japan, and Turkey (obtained from GenBank). Sequences from isolates representing phylogenetic species 1 and 2, as described by Laurence et al. (2014), were also included to provide broader phylogenetic context for $F$. oxysporum $\mathrm{f}$. sp. fragariae isolates within the $F$. oxysporum species complex (FOSC). All $F$. oxysporum f. sp. fragariae isolates obtained for this study grouped with FOSC phylogenetic species 2, and California isolates clustered together with isolates from Japan, although bootstrap support was low (Fig. 1).

Given the absence of polymorphisms in EF- $1 \alpha$ sequences of SCG 1 isolates, a subset of four isolates from this group (GL1080, GL0878, GL1162, and GL1385) was selected for IGS sequencing. The IGS region was also sequenced for all five isolates associated with SCG 2 or SCG 3, three Japanese isolates (MAFF712071, MAFF727510, and MAFF744009), and three nonpathogenic isolates of $F$. oxysporum (GL1702, GL1703, and GL1704) obtained from a field in Monterey County The selected Japanese and nonpathogenic isolates had EF-1 $\alpha$ sequences identical to those of SCG 2 and SCG 3. ML and MP evolutionary models produced trees with identical nodes and similar bootstrap support for this locus. MP analysis of the 2,269-bp IGS sequence alignment revealed 314 parsimony-uninformative and 120 parsimony-informative characters.

Partition homogeneity testing of the EF- $1 \alpha$ and IGS datasets yielded a $P$ value of 0.931 , indicating congruence (Johnson et al. 2001). Therefore, $\mathrm{EF}-1 \alpha$ and IGS sequence alignments were combined and subjected to ML and MP analyses (Fig. 2). Similar to results obtained with the IGS sequence alone, concatenated sequences yielded trees with identical nodes and only minor differences in bootstrap support. In contrast to trees based only on EF- $1 \alpha$ sequences, ML and MP analyses differentiated SCG 2 and SCG 3 with bootstrap support of 79 and 85, respectively. Also, two nonpathogenic isolates clustered with isolates of SCG 2 with high bootstrap support.

Efficacy of PCR and SCG detection methods. Somatic compatibility and PCR test results were in accord for 97\% (191 of 196) of F. oxysporum isolates obtained by intensive sampling of a field in Monterey County; either both were positive or both were negative (Table 3). The diagnostic amplicon was not detected in any isolate that was incompatible with all $F$. oxysporum f. sp. fragariae tester strains. However, five isolates that were negative in the PCR test were compatible with testers representing SCG 1. All five isolates were confirmed to be pathogenic based on development of typical symptoms of Fusarium wilt in inoculated plants (data not shown). Similar results were obtained for isolates recovered from roots of strawberry plants grown in infested soil obtained from Ventura County In all, 66 of 68 isolates (97\%) were either both positive or both negative for PCR and somatic compatibility tests (Table 3). Two isolates were negative in the PCR test but somatically compatible with a tester strain for SCG 1, and both were confirmed to be pathogenic to strawberry (data not shown). No isolates were positive in the PCR test but incompatible with all tester strains (Table 3).

\section{Discussion}

In total, 59 isolates were confirmed as $F$. oxysporum $\mathrm{f}$. sp. fragariae based on development of typical symptoms of Fusarium wilt in inoculated strawberry plants. Phylogenetic analysis of the combined EF- $1 \alpha$ and IGS datasets separated isolates of $F$. oxysporum $\mathrm{f}$. sp. fragariae into three clusters with high bootstrap support. Each cluster corresponded to one of three SCGs (Fig. 2), suggesting that three distinct lineages of $F$. oxysporum f. sp. fragariae occur in California. More extensive testing will be required to determine whether SCGs are associated with quantitative or qualitative differences in virulence to strawberry.

Evidence for a common origin of $F$. oxysporum $\mathrm{f}$. sp. fragariae in California and Japan comes from sequence similarity at IGS and EF-1 $\alpha$ loci in California and Japanese isolates and the presence of a unique diagnostic sequence in both populations. IGS and EF- $1 \alpha$ sequences obtained for MAFF727510 and MAFF712071 are similar to those of SCG 1 and SCG 2, though they did not cluster on the same node (Fig. 2). The diagnostic sequence identified by Suga et al. (2013) for F. oxysporum f. sp. fragariae in Japan targets a noncoding region between two transposons that would therefore be expected to accrue mutations. However, it shares $100 \%$ sequence identity with the amplicon obtained from Californian isolates of $F$. oxysporum $\mathrm{f}$. sp. fragariae. These sequence similarities would be consistent with Californian and Japanese isolates sharing a common origin. However, more extensive sampling and finer resolution of genetic relationships by whole genome sequencing would be required to confirm this.

Sequence differences in the IGS and EF-1 $\alpha$ loci both support resolution of F. oxysporum f. sp. fragariae into distinct clusters. However, the IGS provided a higher proportion of phylogenetically informative characters $(5.2 \%$ of sites) than the EF- $1 \alpha$ locus $(0.03 \%)$. EF- $1 \alpha$ sequences were identical in isolates associated with SCG 2 and SCG 3, whereas IGS sequences were polymorphic within both groups. $F$. oxysporum isolates with identical EF-1 $\alpha$ sequences but dissimilar IGS regions have previously been reported, and this finding supports the use of multilocus

Table 2. Distribution of somatic compatibility groups of Fusarium oxysporum f. sp. fragariae in coastal California counties

\begin{tabular}{lccccc}
\hline & \multicolumn{4}{c}{ Location $^{\mathbf{a}}$} \\
\cline { 2 - 6 } SCG $^{\mathbf{b}}$ & Ventura & Monterey & Santa Cruz & Santa Barbara & San Luis Obispo \\
\hline SCG1 & 28 & 22 & 1 & 3 & 0 \\
SCG2 & 2 & 0 & 0 & 1 & 1 \\
SCG3 & 1 & 0 & 0 & 4 & 0 \\
Total & 31 & 22 & 1 & 6.8 & 1 \\
\% total & 52.5 & 37.3 & 1.7 & & 1.7 \\
\hline
\end{tabular}

a County in California where the field from which isolates were obtained was located.

b Somatic compatibility group. 
sequence data for phylotyping in the FOSC (O'Donnell et al. 2009). However, IGS sequences alone were sufficient for resolving phylogenetic relationships of interest in the present study.

Primers developed by Suga et al. (2013) were reported to produce an amplicon from all Japanese isolates of $F$. oxysporum $\mathrm{f}$. sp. fragariae but also from a small percentage of isolates that were not pathogenic to strawberry. In contrast, we found that all isolates in which the diagnostic amplicon was detected were pathogenic on strawberry, and the amplicon was not detected in any nonpathogenic isolate. However, the amplicon was absent in 4 of 59 isolates of $F$. oxysporum f. sp. fragariae obtained from symptomatic strawberry plants California. All three isolates associated with SCG 2 were PCR negative, suggesting that an entire lineage may lack the diagnostic sequence.

Sequences amplified with the FofraF/FofraR primer pair from California isolates associated with SCG 1 and SCG 3 were both $100 \%$ identical to the sequence published by Suga et al. (2013). Presence of the identical sequence in phylogenetically distinct lineages of $F$. oxysporum f. sp. fragariae could be explained by descent from a common ancestor with no mutations, or horizontal transfer of this sequence, perhaps in association with an accessory chromosome. The latter hypothesis is consistent with experimental evidence of nuclear exchanges, even between isolates that appear to be somatically incompatible (Ma et al. 2010; Shahi et al. 2016). Further indications of horizontal transfer of virulence determinants in the FOSC may be drawn from phylogenetic analysis of genes coding for proteins that are secreted in xylem (SIX genes). For example, within $F$. oxysporum f. sp. lycopersici, SIX gene sequences are identical, or nearly so, despite divergent sequences of phylogenetically informative genes (Van Der Does et al. 2008). Similar findings have been reported in F. oxysporum f. sp. canariensis (Laurence et al. 2015). Although the broader significance of horizontal gene transfer in the FOSC remains to be established, it now seems likely to be an important contributor to the polyphyletic nature of many formae speciales (Ma et al. 2013).

It should be noted that, although Japanese and California isolates of $F$. oxysporum f. sp. fragariae were not observed to be somatically compatible, this does not preclude the possibility of rare fusion events. A pairing reaction is judged to be negative by the absence of aerial mycelium where complementary nit mutants grow together. This does not indicate that anastomosis has not occurred but, rather, that the frequency of hyphal fusion is below the threshold required to supply enough reduced nitrogen to support

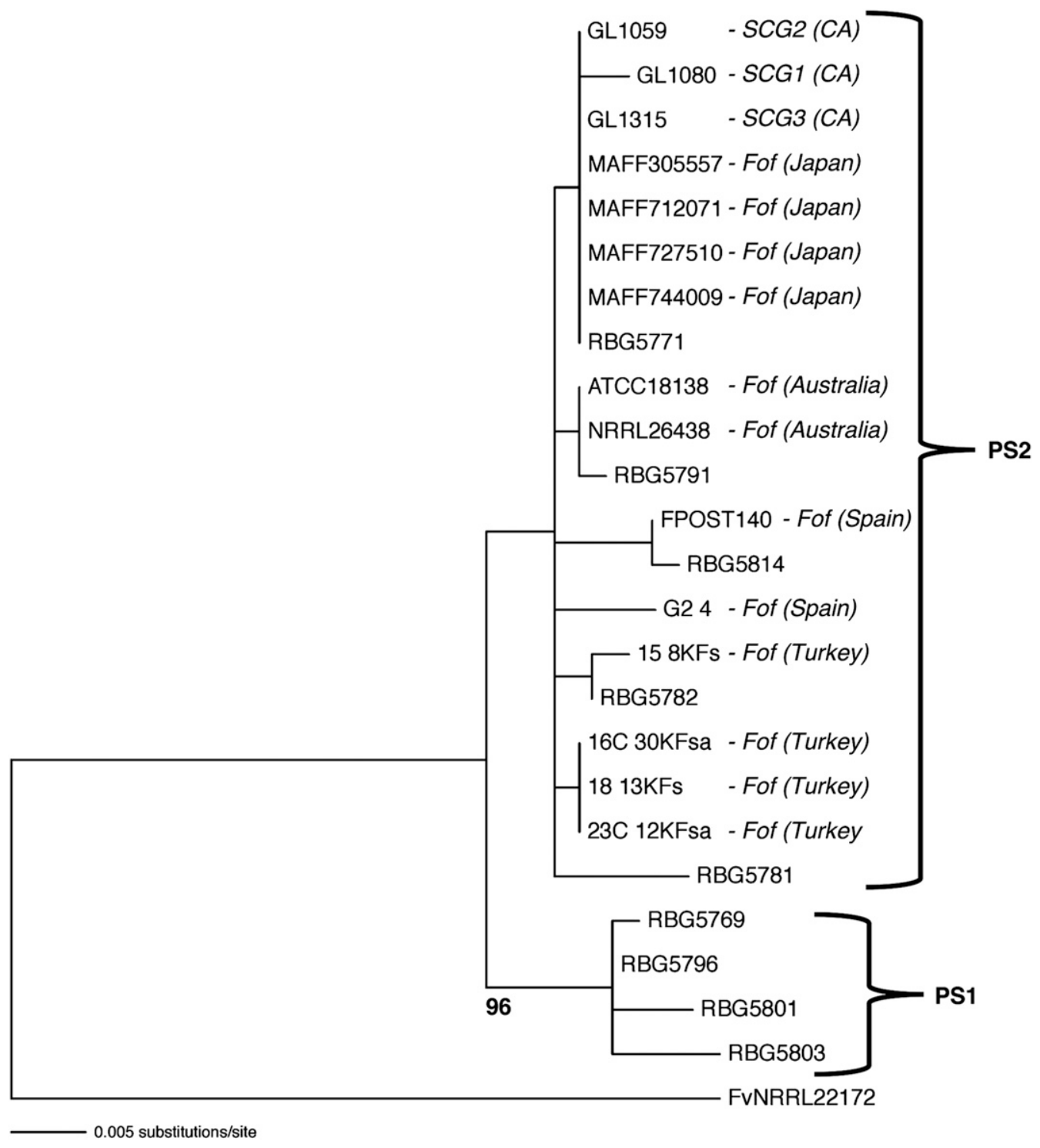

Fig. 1. Maximum-likelihood tree of elongation factor $1-\alpha$ gene sequences from globally distributed isolates of Fusarium oxysporum $\mathrm{f}$. sp. fragariae and representative isolates from phylogenetic species 1 (PS1) and phylogenetic species 2 (PS2) of the F. oxysporum species complex. Codes beginning with "RBG" correspond to reference isolates for PS1 and PS2 whose sequences were reported by Laurence et al. (2014). Bootstrap values are based on 10,000 resamplings, and sequences from F. verticillioides (NRRL 22172) were used for the outgroup species. 
wild-type growth. Thus, isolates regarded as incompatible based on tests using nit mutants may undergo hyphal fusions at some low frequency.

In the collection obtained from soil in Monterey County, only 39\% (77 of 196) of colonies identified as F. oxysporum based on colony morphology were confirmed by subsequent testing to be $F$. oxysporum f. sp. fragariae. In contrast, $94 \%$ (64 of 68 ) of presumptive $F$. oxysporum f. sp. fragariae colonies obtained from roots of strawberry plants grown in infested soil (from Ventura County) were confirmed to be pathogenic on strawberry. The greater utility of colony morphology

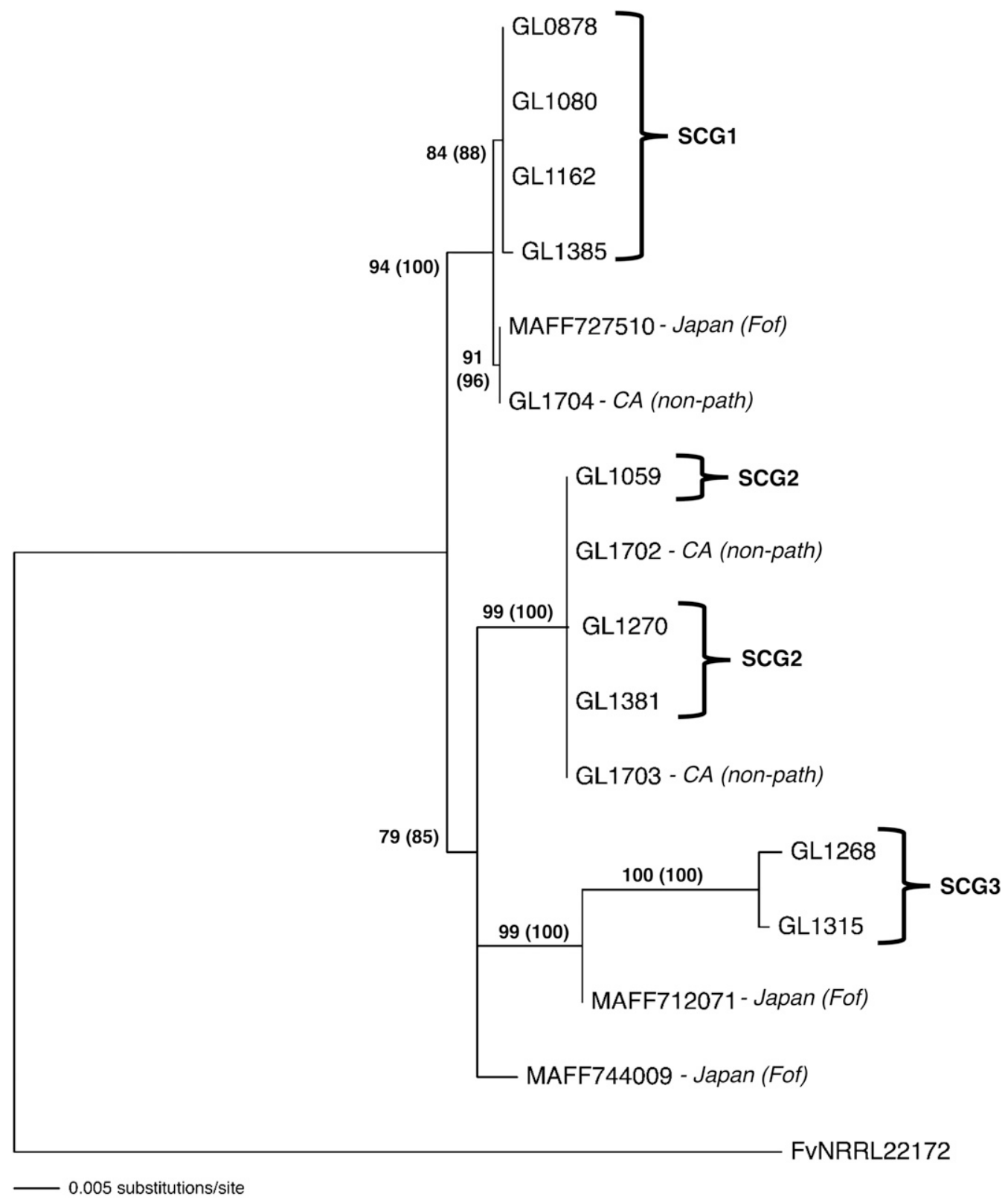

Fig. 2. Maximum-likelihood (ML) tree of concatenated intergenic spacer and elongation factor 1- $\alpha$ gene sequences from Fusarium oxysporum $\mathrm{f}$. $\mathrm{sp}$. fragariae and nonpathogenic isolates of $F$. oxysporum from California, and Japanese isolates of $F$. oxysporum f. sp. fragariae. Significant bootstrap values from ML and maximum-parsimony (MP) analyses (based on 10,000 resamplings) are indicated at each node (MP values are in parentheses). SCG = somatic compatibility group. Outgroup sequences were from $F$. verticillioides NRRL 22172

Table 3. Correspondence between somatic compatibility and the presence of a diagnostic amplicon in isolates of Fusarium oxysporum sampled from two counties in California

\begin{tabular}{lcccr}
\hline Source $^{\mathbf{a}}$ & PCR $+/ \mathbf{S C G}+\mathbf{b}^{\mathbf{b}}$ & PCR-/SCG-c & PCR-/SCG+d & PCR+/SCG-e \\
\hline Monterey County & $72(37 \%)$ & $119(61 \%)$ & $5(3 \%)$ & 0 \\
Ventura County & $62(91 \%)$ & $4(6 \%)$ & $2(3 \%)$ & 0 \\
\hline
\end{tabular}

a County in California where the field from which isolates were obtained was located.

${ }^{\mathrm{b}}$ Diagnostic amplicon was detected by polymerase chain reaction (PCR) and isolate was associated with $F$. oxysporum f. sp. fragariae somatic compatibility group (SCG) 1.

${ }^{\mathrm{c}}$ Diagnostic amplicon was not detected and isolate was not compatible with any $F$. oxysporum f. sp. fragariae tester strain.

${ }^{\mathrm{d}}$ Diagnostic amplicon was not detected but isolate was somatically compatible with a $F$. oxysporum $\mathrm{f}$. sp. fragariae tester strain for SCG 1.

${ }^{\mathrm{e}}$ Diagnostic amplicon was detected but isolate was not compatible with any $F$. oxysporum $\mathrm{f}$. sp. fragariae tester strain. 
for identification purposes in the latter case can be attributed, in part, to fewer nonpathogenic isolates resembling the pathogen on roots than in soil. The extent to which this reflects differences in soil populations at the two locations or selectivity of strawberry roots is not known.

The diagnostic PCR developed by Suga et al. (2013) did not result in amplification for any isolate in SCG 2, suggesting that the target sequence is absent in this clonal lineage. In contrast, an amplicon was obtained from $95.7 \%$ of the 188 isolates associated with SCG 1 and $100 \%$ of isolates in SCG 3. Accordingly, the efficacy of PCR-based detection will be determined by the composition of the pathogen population being sampled. Based on the present assessment, the California population is dominated by SCG 1, which was the only SCG identified at the two sites where pathogen populations were intensively sampled (Table 3). At locations such as these, most or all pathogenic isolates would be detectable by the PCR test whereas, in fields with more diverse populations, some pathogenic isolates may escape detection. Nevertheless, the primers developed by Suga et al. (2013) provide a very useful tool for research and disease diagnosis. A more comprehensive test for detection of $F$. oxysporum $\mathrm{f}$. sp. fragariae may require identification of a sequence directly linked to virulence on strawberry.

\section{Acknowledgments}

This research was supported by grants from the California Strawberry Commission and awards from the Storkan-Hanes-McCaslin Research Foundation. We thank S. Koehler and N. Mitchell for their excellent technical assistance.

\section{Literature Cited}

California Department of Food and Agriculture. 2014. California Agricultural Production Statistics. Online publication. https://www.cdfa.ca.gov/statistics/

Cianchetta, A. N., and Davis, R. M. 2015. Fusarium wilt of cotton: Management strategies. Crop Prot. 73:40-44.

Correll, J. C., Klittich, C. J. R., and Leslie, J. F. 1987. Nitrate nonutilizing mutants of Fusarium oxysporum and their use in vegetative compatibility tests. Phytopathology 77:1640-1646.

Dinler, H., Belioglu, S., and Benlioglu, K. 2016. Occurrence of Fusarium wilt caused by Fusarium oxysporum on strawberry transplants in Aydin Province in Turkey. Australas. Plant Dis. Notes 11:10.

Gordon, T. R., Jacobson, D. J., May, D. M., Tyler, K., and Zink, F. 1990. Fruit yield, disease incidence, and root colonization of hybrid muskmelons resistant to Fusarium wilt. Plant Dis. 74:778-781.

Gordon, T. R., and Okamoto, D. 1991. Vegetative compatibility groupings in a local population of Fusarium oxysporum. Can. J. Bot. 69:168-172.

Hasegawa, M., Kishino, H., and Yano, T. A. 1985. Dating of the human-ape splitting by a molecular clock of mitochondrial DNA. J. Mol. Evol. 22:160-174.

Herron, D. A., Wingfield, M. J., Wingfield, B. D., Rodas, C. A., Marincowitz, S., and Steenkamp, E. T. 2015. Novel taxa in the Fusarium fujikuroi species complex from Pinus spp. Stud. Mycol. 80:131-150.

Jacobson, D. J., and Gordon, T. R. 1988. Vegetative compatibility and selfincompatibility within Fusarium oxysporum f. sp. melonis. Phytopathology 78:668-672.

Johnson, K. P., Drown, D. M., and Clayton, D. H. 2001. A data based parsimony method of co-phylogenetic analysis. Zool. Scr. 30:79-87.

Kettler, T. A., Doran, J. W., and Gilbert, T. W. 2001. Simplified method for soil particle-size determination to accompany soil-quality analyses. Soil Sci. Soc. Am. J. 65:849-852.

Koike, S., and Gordon, T. R. 2015. Management of Fusarium wilt of strawberry. Crop Prot. 73:67-72

Koike, S. T., Kirkpatrick, S. C., and Gordon, T. R. 2009. Fusarium wilt of strawberry caused by Fusarium oxysporum in California. Plant Dis. 93:1077.

Komada, H. 1975. Development of a selective medium for quantitative isolation of Fusarium oxysporum from natural soils. Rev. Plant Prot. Res. 8:114-125.

Larkin, M. A., Blackshields, G., Brown, N. P., Chenna, R., McGettigan, P. A., McWilliam, H., Valentin, F., Wallace, I. M., Wilm, A., Lopez, R., Thompson, J. D., Gibson, T. J., and Higgins, D. G. 2007. ClustalW and ClustalX version 2. Bioinformatics 23:2947-2948
Laurence, M. H., Summerell, B. A., Burgess, L. W., and Liew, E. C. Y. 2014 Genealogical concordance phylogenetic species recognition in the Fusarium oxysporum species complex. Fungal Biol. 118:374-384.

Laurence, M. H., Summerell, B. A., and Liew, E. C. Y. 2015. Fusarium oxysporum f. sp. canariensis: Evidence for horizontal gene transfer of putative pathogenicity genes. Plant Pathol. 64:1068-1075.

Ma, L., Geiser, D. M., Proctor, R. H., Rooney, A. P., O’Donnell, K., Trail, F., Gardiner, D. M., Manners, J. M., and Kazan, K. 2013. Fusarium pathogenomics. Annu. Rev. Microbiol. 67:399-416.

Ma, L.-J., van der Does, H. C., Borkovich, K. A., Coleman, J. J., Daboussi, M.-J., Di Pietro, A., Dufresne, M., Freitag, M., Grabherr, M., Henrissat, B., Houterman, P. M., Kang, S., Shim, W.-B., Woloshuk, C., Xie, X., Xu, J.-R., Antoniw, J. Baker, S. E., Bluhm, B. H., Breakspear, A., Brown, D. W., Butchko, R. A. E., Chapman, S., Coulson, R., Coutinho, P. M., Danchin, E. G. J., Diener, A., Gale, L. R., Gardiner, D. M., Goff, S., Hammond-Kosack, K. E., Hilburn, K., Hua-Van, A., Jonkers, W., Kazan, K., Kodira, C. D., Koehrsen, M., Kumar, L., Lee, Y.-H., Li, L., Manners, J. M., Miranda-Saavedra, D., Mukherjee, M., Park, G., Park, J., Park, S.-Y., Proctor, R. H., Regev, A., Ruiz-Roldan, M. C., Sain, D., Sakthikumar, S., Sykes, S., Schwartz, D. C., Turgeon, B. G., Wapinski, I., Yoder, O., Young, S., Zeng, Q., Zhou, S., Galagan, J., Cuomol, C. A., Kistler, H. C., and Rep, M. 2010. Comparative genomics reveals mobile pathogenicity chromosomes in Fusarium. Nature 464:367-373.

McGovern, R. J. 2015. Management of tomato diseases caused by Fusarium oxysporum. Crop Prot. 73:78-92.

O’Donnell, K., Gueidan, C., Sink, S., Johnston, P. R., Crous, P. W., Glenn, A., Riley, R., Zitomer, N. C., Colyer, P., and Waalwijk, C. 2009. A two-locus DNA sequence database for typing plant and human pathogens within the Fusarium oxysporum species complex. Fungal Genet. Biol. 46:936-948.

O’Donnell, K., Kistler, H. C., Cigelnik, E., and Ploetz, R. C. 1998. Multiple evolutionary origins of the fungus causing Panama disease of banana: Concordant evidence from nuclear and mitochondrial gene genealogies. Proc. Nat. Acad. Sci. USA 95:2044-2049.

Okamoto, H., Fujii, S., Kato, K., and Yoshioka, A. 1970. A new strawberry disease 'Fusarium wilt'. Plant Prot. 24:231-235.

Pastrana, A. M. 2014. Incidence and epidemiology of soilborne fungal pathogens of strawberries in Spain: Development of biotechnological tools and implementation of other control strategies. Ph.D. dissertation, University of Seville, Spain.

Schmale, D. G., and Gordon, T. R. 2003. Variation in susceptibility to pitch canker disease, caused by Fusarium circinatum, in native stands of Pinus muricata. Plant Pathol. 52:720-725.

Schweigkofler, W., O’Donnell, K., and Garbelotto, M. 2004. Detection and quantification of airborne conidia of Fusarium circinatum, the causal agent of Pine Pitch Canker, from two California sites by using a real-time PCR approach combined with a simple spore trapping method. Appl. Environ. Microbiol. 70:3512-3520.

Scott, J. C., Gordon, T. R., Shaw, D. V., and Koike, S. T. 2010. Effect of temperature on severity of Fusarium wilt of lettuce caused by Fusarium oxysporum f. sp. lactucae. Plant Dis. 94:13-17.

Shahi, S., Beerens, B., Bosch, M., Linmans, J., and Rep, M. 2016. Nuclear dynamics and genetic rearrangement in heterokaryotic colonies of Fusarium oxysporum. Fungal Genet. Biol. 91:20-31.

Suga, H., Hirayama, Y., Morishima, M., Suzuki, T., Kageyama, K., and Hyakumachi, M. 2013. Development of PCR primers to identify Fusarium oxysporum f. sp. fragariae. Plant Dis. 97:619-625.

Swofford, D. L. 2002. PAUP*: Phylogenetic Analysis Using Parsimony (* and other methods), version 4. Sinauer Associates, Sunderland, MA.

Tamura, K., Stecher, G., Peterson, D., Filipski, A., and Kumar, S. 2013. MEGA6: Molecular evolutionary genetics analysis version 6.0. Mol. Biol. Evol. 30: $2725-2729$.

USDA Economic Research Service. 2015. Yearbook Tables. Fruit and Tree Nut Data. Online publication. United States Department of Agriculture (USDA). http://www.ers.usda.gov/data-products/fruit-and-tree-nut-data/yearbook-tables. aspx

Van Der Does, C. H., Lievens, B., Claes, L., Houterman, P. M., Cornelissen, B. J. C., and Rep, M. 2008. The presence of a virulence locus discriminates Fusarium oxysporum isolates causing tomato wilt from other isolates. Environ. Microbiol. 10:1475-1485.

Winks, B. L., and Williams, Y. N. 1965. A wilt of strawberry caused by a new form of Fusarium oxysporum. Queensl. J. Agric. Anim. Sci. 22:475-479. 